\title{
Norois
}

Environnement, aménagement, société

\section{Pratiques de nature populaires et écologisation du territoire}

Les effets sociaux de la requalification d'un espace littoral

Working-class Practices Relating to Nature and the Ecologization of Territories.

The Social Effects of the Reclassification of a Coastal Area

\section{Laurence Nicolas}

\section{OpenEdition}

\section{Journals}

\section{Édition électronique}

URL : http://journals.openedition.org/norois/5865

DOI : 10.4000/norois. 5865

ISBN : $978-2-7535-5227-2$

ISSN : 1760-8546

Éditeur

Presses universitaires de Rennes

Édition imprimée

Date de publication : 17 octobre 2016

Pagination : 59-67

ISBN : 978-2-7535-5222-7

ISSN : 0029-182X

Référence électronique

Laurence Nicolas, « Pratiques de nature populaires et écologisation du territoire », Norois [En ligne], 238-239 | 2016, mis en ligne le 17 octobre 2018, consulté le 04 mai 2019. URL : http:// journals.openedition.org/norois/5865; DOI : 10.4000/norois.5865 


Presses
Universitaires
de Rennes

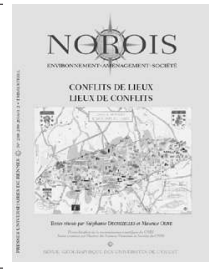

\title{
Pratiques de nature populaires et écologisation du territoire Les effets sociaux de la requalification d'un espace littoral
}

\author{
Working-class Practices Relating to Nature and the Ecologization of Territories \\ The Social Effects of the Reclassification of a Coastal Area
}

\author{
Laurence Nicolas
}

ethnologue indépendante à RESSOURCE \& chercheure associée à l'UMR ESPACE 7300, groupe DESMID 1 rue Parmentier 13200 Arles, France. (Laurence.b.nicolas@wanadoo.fr)

\begin{abstract}
Résumé : Beauduc, plage du littoral camarguais, fait depuis plusieurs décennies l'objet d'une appropriation sociale de l'espace relevant des couches populaires locales. Celles-ci y érigent des cabanons, sans droit ni titre, formant le support de pratiques de sociabilité, de nature et de loisirs et leur multiplication aboutit à la constitution d'un lieu à soi, favorisant l'entre soi. Improbable utopie populaire, cette zone de non droit est également le lieu d'un ressaisissement et d'un épanouissement d'une citoyenneté active. L'action associative et militante des usagers, soutenus localement, permet à ces modes d'habiter le littoral d'être érigés en biens culturels, éventuellement «patrimonialisables ». S’y côtoient, sans toutefois se mélanger, nouveaux usages sportifs, loisirs balnéaires et pratiques plus traditionnelles du territoire de type pêche et cabanon. Ces pratiques sont cependant loin de faire consensus quant à leur légitimité et leur valeur culturelle. Contestées dans leur existence même par l'appareil législatif (loi Littoral), elles sont également largement décriées d'un point de vue environnemental par les instances locales et nationales de protection de la nature. Sévèrement sanctionnée en 2004 par des opérations de démolition menées par le Préfet de région, cette action collective « déviante » est aujourd'hui confrontée à l'arrivée du Conservatoire du littoral. Ce nouvel acteur public amène à une requalification de l'espace, dans laquelle cette action collective peine à prendre place et à faire valoir sa pratique citoyenne, son mode d'habiter le littoral et le rapport à la nature dont elle est porteuse. La question que propose d'examiner cet article est la suivante : quels éléments de distorsion cognitive autour du rapport à la nature bloquent la (re)connaissance de cette citoyenneté active ?
\end{abstract}

\begin{abstract}
For many decades, Beauduc, located on the Camargue coast, has been socially appropriated by the local working classes, who have illegally constructed huts and cabins there, with no property deeds. These vernacular settlements, built for diverse types of activities - sociability, enjoyment of nature and leisure - have proliferated and led to the constitution of a social grouping, in a place where people "keep themselves to themselves". As an unlikely popular Utopia, this "law-free" area is also a place that has seen active citizenship flourish. Activism and association-based activities among users, supported locally, have enabled these dwelling practices in coastal areas to become established as cultural assets that may be considered "heritage-worthy". New sporting activities, seaside leisure pursuits, and more traditional uses of this territory, such as "fishing and enjoying cabin life", all coexist here, albeit separately. These practices are, however, far from consensual in terms of their legitimacy and their cultural value. Their very existence has been challenged by the legislation in place; in addition, these practices are also widely criticized from an environmental standpoint by local and national nature-protection authorities. Severely sanctioned in 2004 by the regional prefect, who ordered the demolition of these cabins, the authors of this "deviant" collective action are now confronted with the arrival of the Conservatoire du Littoral (Coastal Conservation Authority), a new public actor that has set out plans for the reclassification of this territory. Against the backdrop of this public action, local users are now collectively struggling to exercise their citizen practices and maintain their lifestyle in this coastal area and their relationship with nature. This article proposes to examine the following question: what are the elements of cognitive distortion relating to relationships with nature that constitute an obstacle to (re) cognition of this active citizenship?
\end{abstract}


Mots clés : appropriation de l'espace - cabanisation - conflits sociaux - patrimonialisation - littoral camarguais

Keywords: appropriation of space - hutting - social conflicts - heritage - Camargue coast

\section{INTRODUCTION : Au COMMENCEMENT FUT L'APPROPRIATION... DE LA LAGUNE}

Depuis le milieu du Xx $x^{\mathrm{e}}$ siècle, Beauduc, bout de littoral camarguais, isolé et difficile d'accès, situé aux marges d'un espace industriel voué à l'exploitation salicole, est laissé en déshérence aux mains de couches populaires locales. Ces dernières saisissent là, promptement, l'occasion de se bâtir à peu de frais des rêves de cabanes de pêcheurs et de résidences secondaires. Ce faisant, ces « opportunistes de peu » ne se distinguent guère d'autres cas d'appropriation similaire sise au bord des étangs saumâtres du midi de la France (Gourret, 1898; Bataille-Barragué, 1992 ; Baissette, 1996; Collectif Bourdigou, 1979) et plus ou moins destinée à la chasse, à la pêche, à la convivialité et à la villégiature balnéaire. Les cabanons marseillais (Gontier, 1991) témoignent aussi de ce même engouement pour ces abris « sauvages ». L'économie modeste qui va de pair avec ces formes d'appropriation spatiale s'accompagne d'une architecture qui indique généralement de manière assez précise l'origine sociale et culturelle de ses adeptes, qu'ils soient pêcheurs, chasseurs, ouvriers ou paysans, ainsi que l'affectation principale de la pratique qui y prend corps. Elle renseigne également sur l'inscription spatiale et temporelle de ces appropriations de bords de mer et d'étangs. Les cabanes de roseaux renvoient à un monde préindustriel et lagunaire alors que les cabanons marseillais accompagnent l'essor du monde industriel ouvrier et l'émergence du temps du loisir (Corbin, 1995). Un troisième type se rencontre cependant, plus contemporain, reflet d'une société de consommation productrice d'une abondance d'éléments matériels mis au rebut. Ces nouvelles formes architecturales du pauvre, qui correspondent à ce troisième type, se déclinent sur le mode du bidonville. Cette « cabanisation » est caractérisée parfois comme la face cachée de l'urbanisation littorale, relevant du garbage housing (Cadoret et Lavaud-Letilleul, 2013). Mais, en dépit de l'anglicisme utilisé, la connotation dévalorisante qui s'attache à cette technique de construction établie à partir de la récupération de déchets demeure. Beauduc s'inscrit pleinement dans ce troisième type d'appropriation de l'espace.

C'est pourtant bien ce caractère de garbage housing se dégageant de l'ensemble des installations balnéaires de fortune regroupées à Beauduc qui est posé d'emblée comme un des éléments de la dispute dont l'espace est l'objet. La polémique à leur sujet démarre ainsi à une échelle très locale, dès les années 1950, entre les successeurs des cabanes « traditionnelles » de pêcheurs ${ }^{1}$ qui optent pour des matériaux de construction plus «modernes » et l'élite locale soucieuse de l'image du territoire, de sa camarguité (Nicolas, 2008). La matérialité qu'engage le déroulement de la pratique ouvre, ici, le conflit de lieu. Le premier ressort de la dispute est donc d'ordre esthétique, il s'agit d'un jugement de goût et ce jugement est émis à l'échelle locale. Il s'assortit dans la foulée d'un jugement social portant sur la population concernée par ces usages du littoral.

\section{Inventaire des formes matérielles de l'appropriation ou comment fonder une cité idéale...}

L'ensemble des éléments qui forment la base de cette appropriation du littoral est constitué de cabanes édifiées à partir de récupérations diverses effectuées sur les chantiers ou à l'usine dans le cadre du travail, caisses ou planches en bois, en plastique, en acier pour les parois des habitations, rouleaux de feutres et de caoutchouc pour doubler les cloisons ou maintenir le sable alentour, cuves et bidons en plastique pour transporter ou récupérer l'eau de pluie (figure 1)... Les installations humaines s'établissent sur les quelques hauteurs de ce littoral plat, dunes naturelles ou remblaiement de sable. L'habitat y est en constante évolution, «vivant » au gré de l'extension familiale ou amicale ainsi que des opportunités qu’offre le réseau d'échange matériel dont dispose chacun.

1. Les quelques cabanes traditionnelles qui existaient jusque-là ont été détruites pendant la Seconde Guerre mondiale, le littoral, site de débarquement possible des forces alliées est alors occupé et surveillé par l'armée allemande. 
À partir des années 1960-70, caravanes, autobus, fourgons, containers (figure 2) viennent compléter l'inventaire à la Prévert de ces formes matérielles d'occupation et d'appropriation de l'espace. Leur répartition obéit à une logique sociale et familiale qui finalement aboutit à une forme de hiérarchisation sociale des installations et, partant, de leurs occupants. Le quartier de Beauduc plage accueille ainsi les nouveaux arrivants, les plus jeunes, mais aussi ceux optant pour des installations légères et provisoires tandis que le quartier du «village » abrite les anciens, plus âgés et mieux installés dans de coquets cabanons dont une part importante sont bâtis en dur. Le quartier de Beauduc nord constituant une phase intermédiaire, suivant les mêmes critères de classe d'âge et de type d'occupation bâtie.

Trois quartiers d'installations, regroupant près de 450 unités familiales, forment ainsi au tout début du $\mathrm{XXI}^{\mathrm{e}}$ siècle une cité balnéaire populaire et bricolée de toutes pièces (figure 3 - planche III). Les influences matérielles et symboliques dont fait montre cette « cité », bâtie progressivement, sont multiples et révèlent en creux les enjeux sociaux et culturels poursuivis. Elles nous renseignent d'abord sur la prédominance de l'appartenance à un monde ouvrier, qu'il soit d'origine industrielle ou paysanne, qui caractérise les tenants de cette appropriation de la plage de Beauduc. Se saisissant frénétiquement de toutes sortes d'objets et d'éléments matériels mis au rebut, détournés, récupérés, refaçonnés, réappropriés et ainsi prolongés, des familles, des groupes d'amis, issus du vaste pays d'Arles, ouvriers, artisans, commerçants ou paysans, se reconstruisent un monde et viennent passer l'essentiel de leur temps libre (Claeys et Nicolas, 1999).

Cet entre soi, si caractéristique de l'univers des cabanes, aboutit rapidement au développement d'une appropriation « existentielle » (Ripoll et Veschambre, 2005). Outre les ressources naturelles qui viennent agrémenter l'ordinaire des usagers qui les prélèvent via la pêche, la chasse ou la cueillette, l'espace approprié et les installations plus ou moins précaires qui en découlent deviennent aussi l'occasion de nombreux échanges matériels et symboliques qui fortifient liens sociaux et amicaux : «On pourrait considérer qu'il y a processus/stratégie d'appropriation "symbolique" ou "identitaire" de l'espace par un individu ou un groupe quand

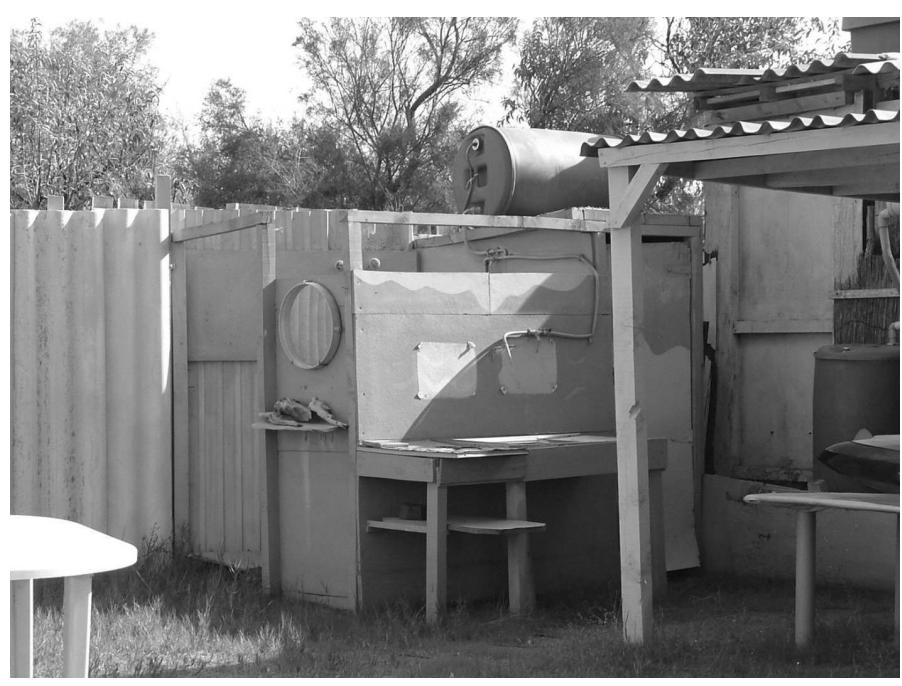

Figure 1 : Partie d'une installation à Beauduc (photo Laurence Nicolas) Part of an installation at Beauduc

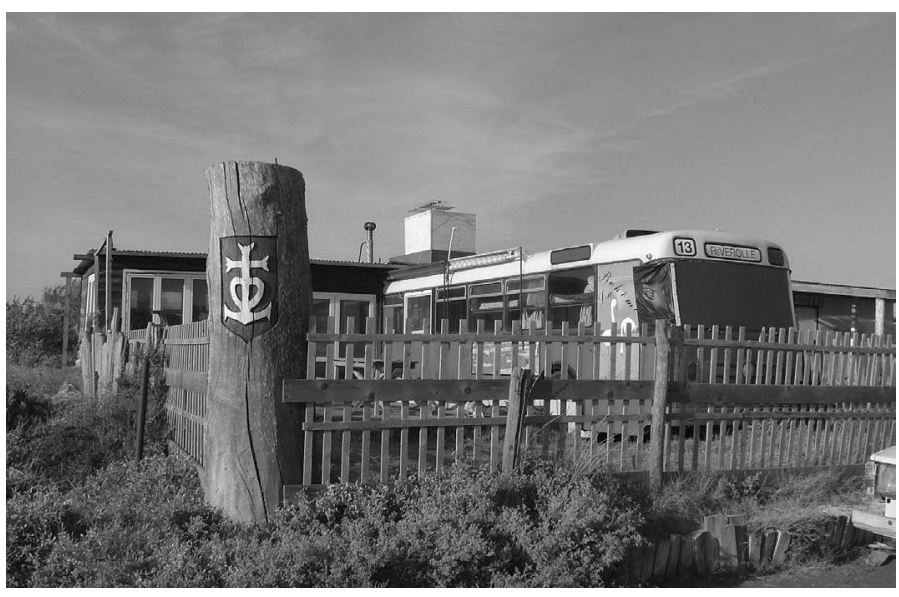

Figure 2 : Type d'installation : le bus cabanon (photo Laurence Nicolas) A unique kind of installation: the "bus cabin"

ses pratiques sociales (marquage, discours, etc.) concourent/visent à la construction et la reconnaissance sociales d'une relation d'association durable, tendant à l'exclusivité et généralement valorisante avec une portion de la surface terrestre. Autrement dit, s'approprier un espace, ce serait ici l'intégrer comme nouvel attribut identitaire pouvant être utilisé comme ressource ou capital symbolique. »(Ripoll, 2005 : 10.)

Cette appropriation correspond en effet à une sorte d'identité alternative, seconde, parfois même exclusive pour certains adeptes, et propice à former le ressort d'une forte mobilisation face au risque d'exclusion des lieux qui menace à tout moment. Elle procure également la possibilité d'une revalorisation 
de soi suivant des critères propres à l'échelle des valeurs en cours dans cet espace approprié, replaçant les pouvoirs du faible au sommet de cette échelle, renversant la donne. Le pêcheur incarne tout particulièrement ces pouvoirs du faible qui donnent accès dans ce cadre à des places sociales convoitées. De l'ordonnance de Colbert de 1681, jusqu'à la loi Littoral de 1986, la seule tolérance d'installation et d'occupation du littoral que prévoit l'appareil législatif est accordée aux « travailleurs » de la mer. Dans ses conditions le pêcheur qui occupe généralement le bas de l'échelle sociale incarne ici la figure originelle et fondatrice de l'appropriation. Il devient ainsi le héros incontesté des lieux, y règne en maître et possesseur de la nature, octroyant des emplacements aux uns et aux autres. Placés sous sa protection, les usagers des lieux retrouvent quant à eux la maîtrise presque totale d'un habitat choisi (dépendant tout de même du réseau d'acquisition et d'échange de différents matériaux et services), reflet de soi, formant le support d'une sociabilité intense, offrant la possibilité de privilégier l'autoconsommation, l'autoproduction et esquissant ainsi un horizon idéel proche d'une sorte d'utopie. Espace pourtant de plus en plus convoité, objet de désir (Corbin, 1988), le littoral devient ici le refuge, la cité idéale et progressivement le symbole des gens de peu (Sansot, 1991).

\section{UN MONDE PRESQUE PARFAIT, «SOUS LA POUBELLE, LA PLAGE "}

Incarnant la figure de l'ancêtre fondateur, le pêcheur ${ }^{2}$, habitant permanent et légitime, occupe à Beauduc le haut du pavé. Il est également le dépositaire d'un ensemble de savoirs et de connaissances concernant le milieu et les ressources naturelles. C'est là, la seule distinction sociale qui opère véritablement tandis que l'ensemble des relations sociales est davantage régi par un fort sentiment de communitas (Turner, 1990; Nicolas, 2008) où l'idéal d'égalité prime. Pourtant résider à Beauduc-plage à Beauduc-Nord ou à Beauducvillage n'équivaut ni au même « standing » ni

2. Malgré l'absence d'infrastructure portuaire, Beauduc compte parmi les stations de pêche les plus réputées pour sa productivité halieutique sur le littoral provençal. On y pratique la pêche au filet en mer et en étang, dite pêche aux petits métiers, avec des embarcations de type "barge " adaptées aux faibles fonds. La pêche à pied aux tellines (donax truncullus) est également pratiquée le long de ce littoral sableux. n’implique un risque similaire. Suite à une forme de tolérance accordée à la fin des années 1960 aux premiers arrivants situés dans le noyau ancien de l'occupation humaine appelé localement le «village » et soumis à un numerus clausus, les suivants qui s'installèrent dans les autres zones atterries de cette lagune ne parvinrent pas à bénéficier du modus vivendi s'appliquant aux premières cabanes et, à partir de 2004, après une décennie de poursuite juridique, furent progressivement chassés.

Les conflits larvés de l'entre soi vont bon train et la quête d'une installation répertoriée anime nombre d'adeptes. En dépit de l'idéal, sinon de l'injonction d'égalité $^{3}$, un clivage s'installe entre ceux qui se prévalent Beauducois et ceux qui sont désignés ironiquement comme les beauduculs ${ }^{4} \ldots$

Par ailleurs, d'autres groupes d'usagers, véliplanchistes, conducteurs de chars à voile, amateurs d'engins motorisés tout terrain, $4 \times 4$, quads, kite-surfer et raveurs sont de plus en plus nombreux et participent aussi à la fabrication du lieu comme symbole de liberté et d'utopie. Ils poursuivent cependant d'autres horizons philosophiques plus proches de la cité éphémère de type «Black rock city » que l'on rencontre dans le désert du Nevada, lors du festival de musique dit de «burning man » et qui s'inscrivent dans des mouvements artistiques et culturels alternatifs. De fréquents conflits d'usage et d'appropriation de l'espace éclatent entre les différents utilisateurs de ce bout littoral, ils traduisent la plupart du temps un rapport à l'espace et à la nature différents. Les uns, souvent issus des couches populaires locales et privilégiant des savoir-faire qui engagent prédation et valorisation des ressources naturelles, bâtissent leur lien à ce qu'ils considèrent comme étant leur " territoire ", via l'incorporation de la nature. Les autres, plutôt originaires de couches urbaines moyennes et supérieures visent davantage une consommation de l'espace plutôt que des ressources naturelles et la contemplation de la nature plutôt que son incorporation.

3. L'injonction d'égalité se manifeste notamment dans l'habitat ou chacun souhaite l'égal de son voisin en termes de « droit » mais aussi d'équipement. La même observance s'applique dans l'accès aux ressources ou bien encore dans la prise de parole publique lors des réunions.

4. Si la communitas abolit temporairement les déterminations sociales et leur hiérachisation elle peut également en produire de nouvelles (Turner 1990 : 193 ; Nicolas $2008: 415$ ). 


\section{Quand le conflit fait naître le lieu, l'impossible patrimonialisation de l'utopie des gratte-plage}

À partir des années 1990, la poursuite judiciaire se précise à l'égard des contrevenants à la loi Littoral de 1986. Certains des cabaniers sont identifiés et astreints à de fortes amendes journalières tant que les lieux ne sont pas remis en leur état initial. Deux restaurants de poissons érigés par des pêcheurs professionnels locaux sont venus s'ajouter depuis une dizaine d'années à l'ensemble des installations sauvages. Rapidement devenus des établissements branchés et underground de Camargue, ils reçoivent une clientèle huppée et quelques célébrités qui, émus par la situation des "gens de Beauduc", mettent en place un comité de soutien. L'argent récolté dans le cadre de ce comité de soutien permet de payer un avocat chargé de la défense des contrevenants qui portera le dossier jusqu'au Conseil d'État.

Parallèlement à ces actions en justice, la résistance s'organise à l'échelle même de Beauduc. Une charte est rédigée par les associations d'usagers présentes sur les lieux ${ }^{5}$, qui prétend répondre point par point à l'ensemble des «reproches » qui leur sont adressés : insécurité, pollution, illégitimité, prolifération et esthétique... Dans les années 2000, l'autogestion des lieux par ses habitants, ponctuels, réguliers ou permanents, s'intensifie. Les installations font l'objet d'un «embellissement » notable. Celui-ci passe par l'amélioration du confort (toilettes sèches, panneaux solaires et éoliennes) et par l'expression plus appuyée du style "beauducois ", érigé en esthétique propre, pour les constructions.

L'un après l'autre, les problèmes que soulève cette occupation humaine sont examinés et pour la plupart résolus par des actions concrètes et citoyennes parfois très audacieuses. Les solutions pratiques font écho au fonctionnement du lieu, puisées dans le registre de la récupération et de l'échange. Ainsi en va-t-il d'une cuve d'un camion-citerne recyclée en fosse d'aisance collective ou encore d'engins reconvertis en camions de pompiers, mais la plus hardie de toutes concerne l'accès à cet espace littoral

5. L'une des associations regroupe la centaine de cabanes «tolérées » tandis qu'une deuxième représente les caravanes et les cabanons non répertoriés, une troisième enfin, née du comité de soutien fondé pour faire face aux poursuites judiciaires, fut créée afin de fédérer l'ensemble des usagers et pratiques du site. que nécessite l'entretien coûteux et incessant d'une piste à peine carrossable. Longue d'une douzaine de kilomètres et empruntant pour partie une digue à la mer interdite à la circulation automobile, la piste fait pourtant l'objet de réfections confiées à des entreprises locales et « complices » qui effectuent les travaux pour des sommes dérisoires si on les compare aux montants qu'elles auraient coûté «officiellement » aux collectivités locales qui en auraient commandé les travaux.

Pour les associations beauducoises cependant, depuis plus de dix ans, les montants annuels sont considérables ${ }^{6}$. Pour récolter les fonds les « gens de Beauduc » vendent des tee-shirts portant le logo associatif, organisent des repas estivaux réunissant de 200 à 400 personnes (figure 4) et des collectes ponctuelles sur la piste lors des week-ends où la fréquentation est la plus importante...

Recourant ainsi à des fonctionnements de plus en plus hardis pour se défendre, les associations sortent du cénacle étroit de leur espace « existentiel » et portent leur étendard hors la plage, que ce soit en empruntant la voie juridique ou en exprimant leur désaccord dans l'espace public comme lors de manifestations organisées dans la ville d'Arles dont les cortèges s'entourent d'une large partie des édiles locaux. Elles le font également à travers la médiatisation dont fait l'objet le conflit, se saisissant

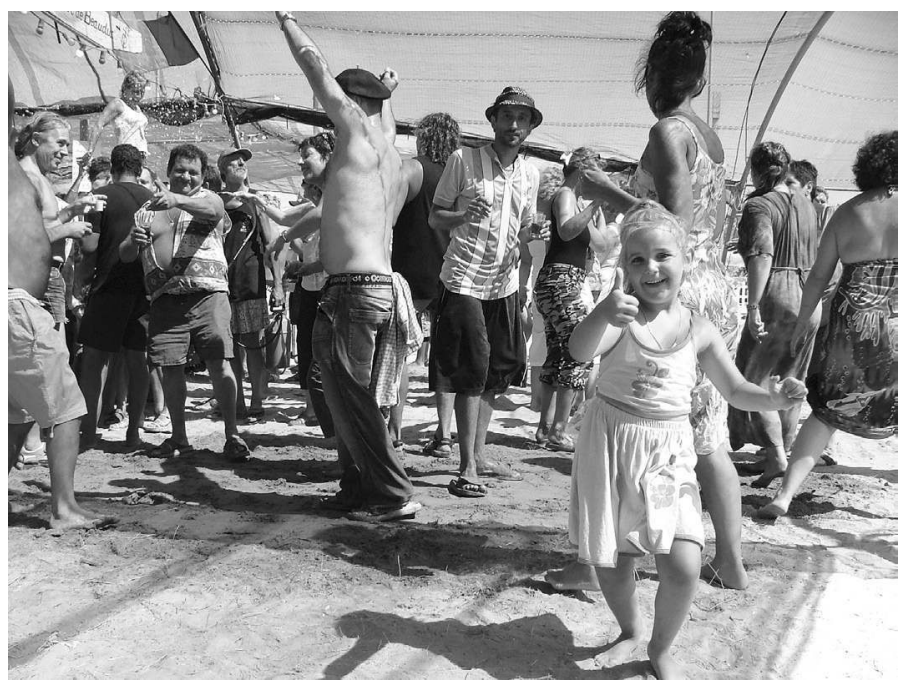

Figure 4 : Fête à Beauduc (photo Laurence Nicolas) A party at Beauduc

6. Environ 20000 euros par an sont nécessaires à l'entretien de la piste. 
d'une popularité grandissante qui en fera l'un des marronniers de l'été jusqu'à aujourd'hui. Bref, le pêcheur, le cabanier, personnifiant jusque-là les pouvoirs du faible (Turner, 1990), finissent par incarner à travers Beauduc, et à la faveur de l'enhardissement de son appropriation, un symbole de liberté et d'utopie, un patrimoine «vivant » qui peine toutefois à faire consensus. Le phénomène de cabanisation beauducoise porte encore le stigmate des origines et si de nombreux intellectuels, architectes et artistes s'émerveillent encore en invoquant le lieu, d'autres, écologues ou préfets, décrient toujours avec force l'horreur de ces constructions et la totale illégitimité dont elles font preuve à leurs yeux.

Il n'est pas étonnant que les deux figures d'opposition ou de dénégation de la valeur «patrimoniale » ou à tout le moins «culturelle» de cette utopie populaire soit précisément le fait de fonctionnaires d'État ou de représentants de la protection de la nature, car la question du rapport à la nature et celle relative à la légitimité de cette appropriation du littoral ne forment peut-être qu'une seule et même question. Ce clivage autour de la "valeur » patrimoniale de ces pratiques mais aussi autour de leur légitimité semble résulter d'une confrontation entre des regards divergents, des perceptions discordantes, relevant tout à la fois de jugements esthétiques, moraux et sociaux et où peinent à s'exprimer et $a$ fortiori se faire (re)connaître des formes particulières, car jugées trop désordonnées, de rapport à la nature? Les « gratte-plage », sobriquet collectif dont s'affublent ironiquement les gens de Beauduc (Nicolas, 2008) se caractérisent pourtant par une forte proximité avec la nature et une faible médiation technique (Barthélémy et Nicolas, 2002) lors de l'acquisition des ressources, privilégiant un véritable corps-à-corps dont témoigne ce surnom d'hommes-fouisseurs.

\section{La mise en ordre des "éco-vilains ", de la démolition à la normalisation}

En 2003, la Directive Territoriale d'Aménagement des Bouches-du-Rhône inscrit pourtant la reconnaissance du hameau des sablons comme une particularité de l'occupation du littoral arlésien. Il s'agit de la partie la plus ancienne des cabanes faisant déjà l'objet d'une tolérance. Cette "labellisation » d'une partie des cabanes de Beauduc peut être apparentée à une forme de patrimonialisation, mais à peine un an plus tard, une opération de démolition de dix-sept installations et des deux restaurants de poissons (cf. figure 3 - planche III), organisée par la préfecture de région Provence-Alpes-Côte-d'Azur, vient brutalement contrebalancer cette timide tentative (figure 5).

L'arrivée d'un nouvel acteur public va en quelque sorte cristalliser cette confrontation entre regards divergents sur ce type de pratique et ouvrir progressivement la voie de la normalisation via l'écologisation du territoire.

À partir de 2008, en effet, la déprise industrielle de l'exploitation salicole qui jouxte le littoral sudest camarguais donne lieu à une succession de ventes foncières à destination du Conservatoire du Littoral. Diminuant sa production et optant pour une nouvelle technique de fabrication du sel pouvant s'effectuer sur de plus petites surfaces, la Compagnie des Salins du Midi vend 6000 hectares au Conservatoire du littoral qui ambitionne d'y accompagner un retour progressif à l'état naturel des étangs de pré-concentration du sel, jusque-là endigués. L'enjeu écologique est d'importance et consiste à reconnecter sur le plan hydraulique cette partie sud-est du territoire rhodanien, cloisonnée pour l'exploitation salicole, à l'ensemble du delta et à permettre ainsi les échanges biologiques entre les étangs centraux et littoraux. Il s'agit aussi de réintroduire une dynamique naturelle dans un delta entièrement artificialisé (Picon, 2008) en laissant peu à peu la lagune se dépoldériser.

La mission est confiée à plusieurs gestionnaires de nature locaux : le Parc naturel régional de Camargue (PNRC), le centre de recherches pour la conservation des zones humides de la Tour du Valat et la Réserve nationale de Camargue. Jusque-là tenues à l'écart de cette partie du territoire ces institutions locales de protection et de gestion de la nature voient là l'occasion d'une importante opération de renaturation de l'espace et sont, dans un premier temps, chargées d'effectuer un ensemble de diagnostics écologiques et de suivis scientifiques relatifs à l'évolution majeure que subit la lagune de Beauduc.

En effet, l'arrêt de la production de sel change radicalement le milieu : assèchement des étangs endigués - jusque-là avivés artificiellement par une station de pompage pour la production de sel diminution d'une partie de l'avifaune, modification 
de la flore. Ce changement paysager d'importance provoque inquiétude et étonnement au sein des populations locales, tant celle des usagers du site de Beauduc que celle ouvrière, de Salin de Giraud, localité voisine née de l'industrie du sel (Guyonnet et al., 1988; 2008), qui y voit la fin d'un monde familier. La renaturation en cours est fortement critiquée, mal comprise d'autant qu'elle s'accompagne d'un encadrement des usages et d'une forte limitation d'accès à certains espaces jusque-là totalement ouverts à tous. Par ailleurs, l'entretien des digues frontales à la mer mises en place par l'industrie du sel est abandonné, provoquant rapidement des brèches qui inquiètent également les populations locales, voyant là une perte d'intégrité territoriale et un risque accru de submersion marine (Allouche et al., 2014; Nicolas et al., 2015).

L'arrivée du Conservatoire du littoral à Beauduc se traduit rapidement par la ferme volonté d'organiser les usages qui se déploient en tous sens sur cette bande de rivage. La circulation automobile est interdite en 2011 sur la Pointe de Beauduc où un arrêté de biotope est à l'étude qui place en défens une part importante de l'appropriation de l'espace par les différents usages balnéaires, dont le camping sauvage et la pratique du kite-surfing. Un arrêté municipal aboutit à la mise en place d'un gabarit sur la piste qui conduit au site afin d'empêcher l'accès aux camions, gros fourgons et caravanes, il vise également la réorganisation et la répartition de l'ensemble des usages mais sera invalidé en 2012 par une forte mobilisation des écoles de kite-surfing qui soulèvent un vice de forme dans son application.

Cependant la normalisation des usages se poursuit, relayée en 2013 par l'arrêté de biotope ${ }^{7}$ et conduite par le Conservatoire du Littoral qui met en place avec les gestionnaires locaux des équipes d'éco-gardes qui sillonnent le littoral, veillent au respect des nouvelles mesures et procèdent à des délimitations de l'espace, avec la mise en place d'une nouvelle signalétique, de pieux, de barrières et de palissades visant à barrer l'accès ou à canaliser les cheminements erratiques. Ces différentes actions techniques constituent les premières modalités de désappropriation de l'espace. L'accès automobile est cependant encore autorisé sur certains secteurs, notamment pour parvenir aux quartiers

7. L'arrêté préfectoral de création d'une zone de protection de biotope sur la Pointe de Beauduc couvre environ 500 hectares.

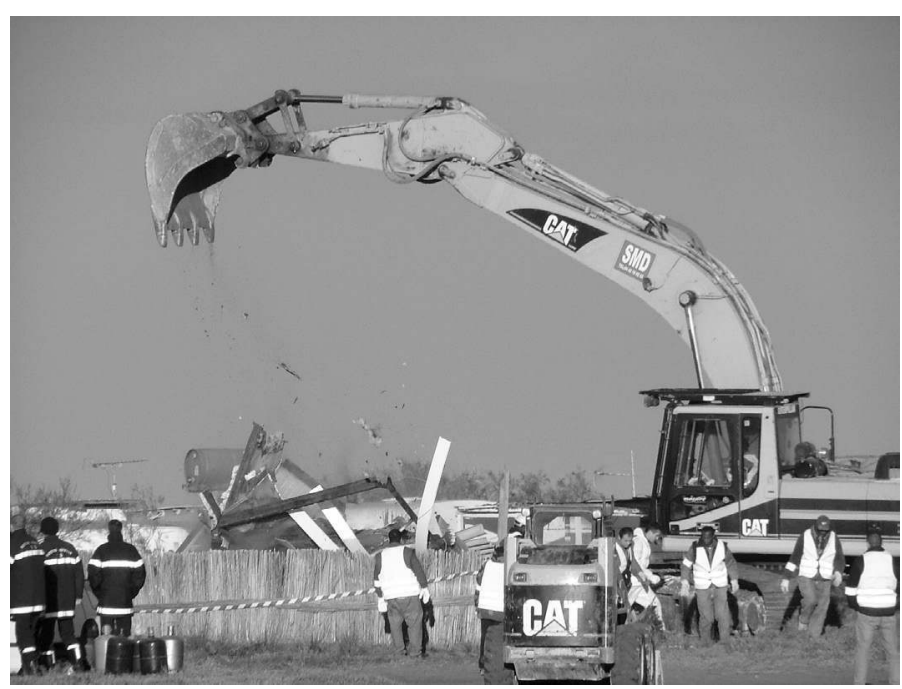

Figure 5 : Opération de démolition d'une partie des installations à Beauduc en fin novembre 2004 (photo Laurence Nicolas)

Demolition of a section of cabins at Beauduc in late November 2004

d'installations humaines. L'emprise spatiale qu'ils constituent demeure une enclave, dont reste encore propriétaire la Compagnie des salins du midi, à l'intérieur des 6585 hectares du Conservatoire du littoral. Cette enclave, perçue comme peau de chagrin par les habitants, est parallèlement l'objet d'une normalisation en cours à travers l'élaboration d'une " charte du cabanon », visant à fixer un ensemble de prescriptions dans divers domaines comme l'esthétique, l'assainissement, la sécurité, les conditions d'entretien des installations...

Le travail est mené par le PNRC en concertation avec les associations de cabanonniers. Sur le terrain, les relations établies avec les éco-gardes s'inscrivent dans une délicate éducation à l'environnement, mal perçue des usagers, même si l'ensemble des nouvelles interdictions est globalement respecté. Car qu'il s'agisse de l'élaboration de la charte du cabanon ou de la sensibilisation à la protection de la nature, le rapport qui s'institue s'apparente inévitablement à celui du maître à l'élève. Ainsi, certaines des injonctions prescrites dans la charte étaient déjà le fait de pratiques existantes et incorporées depuis longtemps par les habitants, objet d'une transmission très pratique et surtout quotidienne auprès des enfants, qu'elles concernent les équipements pour produire de l'énergie (panneaux solaires et éoliennes), la récupération d'eau, les savoir-faire habitants dans l'adaptation au milieu littoral, l'acquisition des ressources naturelles. 
Au cours de l'une des réunions d'élaboration du document, un représentant des cabaniers s'indigne à la lecture d'une recommandation qui précise que l'eau récupérée dans les citernes dont sont équipées toutes les installations ne doit pas être consommée : «On nous fait la leçon comme si on était des gamins à l'école, comme si on était des idiots ou des ignorants, comme si on donnait l'eau de la citerne aux petits dans le biberon! » Les savoirs naturalistes populaires, ainsi que ceux relatifs au milieu, qu'ils concernent son évolution ou l'adaptation à celui-ci - à l'heure où l'on déplore la perte présumée de la culture du risque, au sein des populations, notamment urbaines, lorsqu'elles sont exposées aux changements globaux - sont également ignorés (Barthélémy, 2013; Allouche et Nicolas, 2014). Leur valorisation pourrait cependant enrichir les savoirs et les suivis scientifiques et savants qui sont déployés actuellement sur cet espace en profonde mutation et améliorer nettement la perception des opérations de renaturation en cours de la part des locaux. Mais ces derniers en lieu et place d'être perçus comme d'éventuelles «sentinelles » du littoral, dépositaires de savoirs sensibles, de savoirfaire et de savoir être, attachés affectivement au territoire, sont plutôt désignés comme des « écovilains $^{8}$ », terme emprunté à Marie Roué (2013: 267), exerçant une pression sur le milieu.

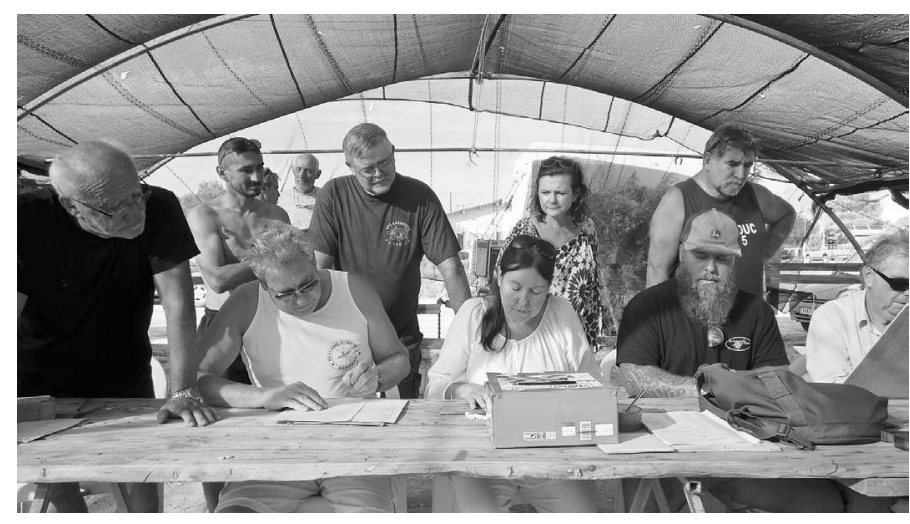

Figure 6 : Dépouillement du vote pour l'élection d'un nouveau conseil d'administration effectué sous la vigilance des adhérents (août 2015, photo Laurence Nicolas)

Counting the votes for the election of a new management board, under the watchful eyes of members (August 2015)

8. L'auteur compare deux processus de patrimonialisation, l'un concernant le site de Laponia au nord de la Suède, l'autre situé dans les Causses en Cévennes, et s'intéresse à la participation des populations locales à cette patrimonialisation en examinant les conditions d'une cogestion entre les décideurs et gestionnaires - plutôt enclins à protéger suivant des critères exclusivement naturalistes - et les autochtones.
À la décharge cependant des naturalistes locaux, gestionnaires, écologues, éco-gardes, les termes du conflit dont la requalification du lieu est l'enjeu restent larvés, non-dits, ou sur un mode agressif et maladroit de la part des «éco-vilains » peu habitués à faire valoir leurs connaissances vernaculaires. Or, le mépris dont ils pensent être l'objet les agace et redouble leur agressivité au point que celle-ci se traduit aussi, peut-être même surtout, dans l'entre soi et notamment dans le cadre associatif.

\section{L'INSUPPORTABLE RELATION DU MAÎTRE À L'ÉLÈVE}

Des trois associations en présence, que nous avons mentionnées précédemment, la plus ancienne d'entre elles avait toujours pris grand soin de choisir ses représentants parmi les cabaniers les plus «assis » socialement (Nicolas, 2008), sachant s'exprimer oralement pour faire face aux négociations avec les autorités et garantir la défense des usagers. Le dernier en date fut un instituteur retraité, peu intégré au groupe et peu rompu à l'exercice de la parole en public, en dépit de son activité professionnelle passée. Il fut cependant poussé par ses prédécesseurs dans la fonction de président de l'association et l'assura pendant une dizaine d'années. Soupçonné de mener des négociations en solo et de prendre des décisions sans en référer aux adhérents, il fut très récemment démis collectivement de ses fonctions dans le cadre d'une assemblée générale extraordinaire tendue (figure 6), à laquelle participèrent la presque totalité des cabaniers. Son successeur, élu à plus de $85 \%$ des voix exprimées, est aujourd'hui un pêcheur professionnel, habitant et figure emblématique du lieu, sans doute choisi comme le plus sûr représentant de ces savoirs et de ce lien au territoire dont sont perçus, sans doute encore confusément, l'importance et l'urgence de leur expression sociale. Cristallisée au plus profond de l'entre soi, la relation du maître à l'élève symbolisée par le statut social du président sortant semblait soudain devenue insupportable, «il nous prend pour ses élèves » répètent à l'envi ses opposants pour justifier sa destitution et cette sensation semble faire écho aux différents rapports sociaux entretenus entre les «éco-vilains » et les nouveaux gestionnaires de l'espace à Beauduc. 


\section{VERS LA PERTE D'UN LIEN AU MILIEU?}

Jusque-là maîtres et possesseurs des lieux, les Beauducois se trouvent aujourd'hui confrontés aux exigences environnementalistes des nouveaux gestionnaires. La restriction de leurs usages, leur cantonnement spatial et la non prise en compte des savoirs profanes qui réglaient auparavant leur rapport à la nature - jugés non pertinents par la gestion environnementaliste de cet espace littoral - ont insidieusement fait naître chez eux une vision négative et pessimiste des choses concernant leur inscription territoriale et son devenir. Une perte d'objectivité s'observe alors qui concourt à une vision inexacte du monde et s'apparente à une distorsion cognitive (Beck, 1967). Le président-instituteur, que l'on avait poussé à des responsabilités associatives, est tout à coup suspecté d'adopter la posture des gestionnaires avec lesquels les relations sociales sont vécues comme étant entachées de mépris social. La façon de traiter l'information, constamment «polluée » par le sentiment de disqualification de la pratique, génère des tensions et aboutit à une mise en berne des activités d'intendance du milieu par les habitants. Les effets sociaux de la requalification de l'espace pourraient ainsi, bientôt, se traduire par la perte du lien tissé avec le milieu par les Beauducois depuis plusieurs générations.

\section{Bibliographie}

Allouche A., Nicolas L. (dir.), 2014. Adaptation aux changements globaux dans la Réserve de Biosphère "Camargue Grand Delta », Rapport final CAMADAPT, programme LITEAU, Ministère de l'Environnement, UMR ESPACE 7300, 176 p., (non publié).

Baissette G., 1996. L'étang de l'or, Sète, Nouvelles presses du Languedoc, $241 \mathrm{p}$.

Barthelemy C., 2013. La pêche amateur au fil du Rhône et de l'histoire, Paris, l'Harmattan, 174 p.

Barthelemy C., Nicolas L., 2002. Pêcheurs amateurs du fleuve à la mer. Des pratiques ordinaires de nature au défi de la gestion environnementale, Europaea, Journal des Europanéistes I - 2, anno VIII, Naturalia, p. 283-301.

Bataille-Barragué R., 1992. L'habitat en roseau traditionnel. Les baraques de sanhils des pêcheurs roussillonnais, Direction régionales des affaires culturelles du Languedoc-Roussillon, rapport dactylographié (non publié).

Beck A. T., 1967. Depression: Causes and treatment, Philadelphia, University of Pennsylvania Press, 370 p.
Cadoret A., Lavaud-Letilleul V., 2013. Des « cabanes » à la «cabanisation » : la face cachée de l'urbanisation sur le littoral du Languedoc-Roussillon, Espaces, populations, sociétés, 1-2, p. 125-139.

Claeys-Mekdade C., Nicolas L., 1999. De la plage appropriée à la plage patrimonialisée, analyse de pratiques balnéaires « déviantes » sur le littoral camarguais : l'exemple de Piémanson et Beauduc, Méditerranée, n 4, p. 27-34.

Collectif, 1979. Bourdigou, le massacre d'un village populaire, Marcevol, éd. du Chiendent, 194 p.

Corbin A., 1988. Le territoire du vide. L'Occident et le désir de rivage, 1750-1840, Paris, Aubier, 411 p.

Corbin A. (dir.), 1995. L'avènement des loisirs, 1850-1960, Paris, Aubier, $471 \mathrm{p}$.

Gontier C., 1991. Le cabanon marseillais, images et pratiques, Marseille, Cerfise, 303 p.

Gourret P., 1897. Les étangs saumâtres du midi de la France et leurs pêcheries, Annales du Musée d'Histoire Naturelle de Marseille - Zoologie - Tome V, Marseille, 386 p.

Guyonnet M.-H., RaVis-giordani G., Villain N., 1988. Salinde-Girand: logique industrielle, logique sociale, Rapport pour la Mission du patrimoine ethnologique et le ministère de la Culture, $161 \mathrm{p}$.

Guyonnet M.-H., 2008. L'Empire du sel, Salin-de-Giraud: une ville ouvrière en Camargue, Manosque, éd. Bec en l'air, $132 \mathrm{p}$.

Nicolas L., 2008. Beauduc, l'utopie des gratte-plage - Ethnographie d'une communauté de cabaniers sur le littoral camarguais, Marseille, IEM éditions, 447 p.

Nicolas L., Allouche A., Dervieux A., Mesleard F., Sandoz A., 2015. Gestion participative des paysages : construction d'une ressource culturelle pour l'appropriation des enjeux de biodiversité?, in Luginbühl Y. (dir.), Biodiversité, paysage et cadre de vie. La démocratie en pratique, Paris, Victoires Éditions, p. 35-54.

Picon B., 2008. L'espace et le temps en Camargue, Arles, Actes Sud, $301 \mathrm{p}$.

Ripoll F., Veschambre V. (dir.), 2005. L'appropriation de l'espace. Sur la dimension spatiale des inégalités sociales et des rapports de pouvoir, Norois, n ${ }^{\circ} 195,117$ p.

Ripoll F., Veschambre V., 2005. L'appropriation de l'espace comme problématique, Norois, $\mathrm{n}^{\circ}$ 195, p. 7-15.

Ripoll F., 2005. S'approprier l'espace... ou contester son appropriation? Une vue des mouvements sociaux contemporains, Norois, $\mathrm{n}^{\circ} 195$, p. 29-42.

Roué M., 2013. Paysages culturels et gouvernance : nouveau contrat social ou retour du refoulé, in LuginbüHL Y., TeRRASSON D. (dir.), Paysage et développement durable, Paris, Quæ, p. 261-273.

SAnsot P., 1991. Les gens de peu, Paris, PUF, 223 p.

Turner V., 1990, Le phénomène rituel - Structure et contre structure, PUF, Paris, 206 p. 


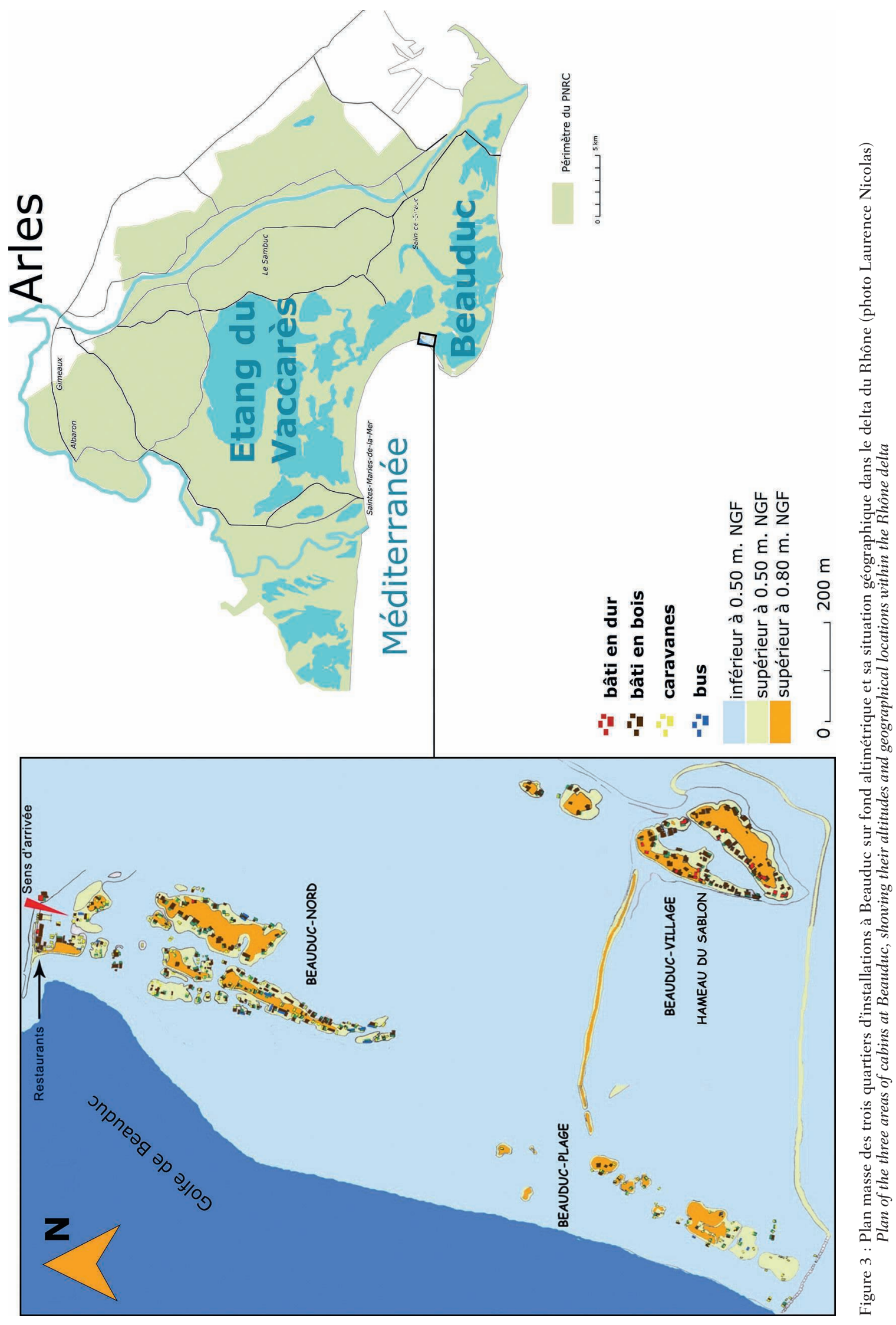

